\title{
Empyème sous-dural post-traumatique
}

\section{Subdural post-traumatic empyema}

\author{
C. Simon $\cdot$ Q. Montaud \\ Reçu le 24 mai 2019; accepté le 2 octobre 2019 \\ (C) SFMU et Lavoisier SAS 2019
}

Un homme de 64 ans, hypertendu et éthylique chronique, consulte aux urgences pour des céphalées intenses et des vomissements évoluant depuis $48 \mathrm{~h}$ malgré une automédication par anti-inflammatoire non stéroïdien et triptan. Il est sujet à des chutes à répétitions dont la dernière, survenue quelques semaines auparavant, a nécessité une consultation aux urgences et un scanner cérébral sans anomalie. Les céphalées sont intenses, pulsatiles, en casque avec une irradiation cervicale. On ne retrouve ni fièvre, ni raideur de nuque et l'examen neurologique initial est normal. Le bilan biologique retrouve un syndrome inflammatoire majeur (CRP $300 \mathrm{mg} / \mathrm{L}$ et hyperleucocytose à $25 \mathrm{G} / \mathrm{L}$ ) motivant la réalisation d'un scanner cérébral sans puis avec injection de produit de contraste. Il apparaît un décollement craniocéphalique droit sous-dural avec déviation de la ligne médiane (Fig. 1A) ainsi qu'une sinusite maxillaire. Le diagnostic d'empyème cérébral est retenu devant un rehaussement de la dure-mère en regard de la collection (Figs 1B,1C). Le patient est transféré en urgence vers le centre de neurochirurgie de référence suite à une dégradation neurologique marquée par des phases d'agitation et de somnolence. Il n'est pas instauré d'antibiothérapie ni de corticothérapie à la demande des neurochirurgiens qui préconisent la réalisation de prélèvements bactériologiques rapides pour une antibiothérapie adaptée. Durant le transfert, il présente une crise comitiale partielle résolutive sous clonazepam suivie d'une absence de retour à la conscience et un coma avec un score de 8 sur l'échelle de Glasgow. Il est pris en charge immédiatement au bloc opératoire pour une ponctionaspiration par trou de trépan. Il a été décidé de réaliser la ponction lombaire en postopératoire pour éviter les risques d'engagement. Celle-ci retrouve un liquide trouble avec

\footnotetext{
C. Simon $(\square)$

Service d'accueil des Urgences-SMUR, Hôpital Manchester, 45, avenue de Manchester, F-08000 Charleville-Mézières, France e-mail : cam08.simon@hotmail.fr

Q. Montaud

Service d'accueil des Urgences, Hôpital Manchester,

45, avenue de Manchester, F-08000 Charleville-Mézières, France
}

1670 leucocytes $/ \mathrm{mm}^{3}$ à prédominance de polynucléaires neutrophiles. Les antigènes pneumocoques réalisés sur le liquide céphalo-rachidien reviennent positifs. Le germe est confirmé par l'examen direct, la culture ainsi que les hémocultures qui avaient été réalisées aux urgences. Une antibiothérapie par céfotaxime est initiée, de même qu'une corticothérapie par déxaméthasone. Les suites opératoires sont marquées par un bref recours à l'osmothérapie et le patient sort de réanimation à huit jours sans séquelles. Les investigations à la recherche d'une porte d'entrée infectieuse ont retenu l'hypothèse de brèche durale post-chute dans un contexte d'éthylisme chronique.

L'empyème cérébral extra- ou sous-dural, est un diagnostic rare en médecine d'urgence. Il est le plus souvent d'origine infectieuse dentaire ou oto-rhino-laryngologique [1,2]. La présentation clinique est principalement marquée par des signes d'hypertension intracrânienne, des crises comitiales, des déficits neurologiques et des troubles de la conscience. Il existe en général un syndrome inflammatoire mais la fièvre peut être absente au stade initial ce qui favorise l'errance diagnostique [2]. La ponction lombaire est primordiale pour le diagnostic bactériologique ainsi que pour le pronostic qui est plus défavorable si une méningite s'y associe. L'enjeu principal de l'imagerie, et en l'occurrence de l'IRM ou du scanner cérébral injecté, est d'établir rapidement le diagnostic afin d'organiser la prise en charge chirurgicale ainsi que d'éliminer les diagnostics différentiels tels que les hématomes sous-duraux et extra-duraux, les abcès et les tumeurs cérébrales. Ces examens visent aussi à rechercher une porte d'entrée infectieuse et un foyer profond latent. La plupart des cas rapportés dans la littérature relatent des complications sinusiennes, otologiques et plus rarement méningée [1,2]. La surinfection d'hématome cérébral post-traumatique est plus rare. Dans cette observation, l'imagerie met en évidence une sinusite maxillaire asymptomatique qui n'a peut-être aucun lien avec l'empyème cérébral. Le pronostic à court terme dépend essentiellement du délai de prise en charge. La conduite thérapeutique à tenir associe d'une part une antibiothérapie à large spectre à forte dose qui doit être débutée sans délai après réalisation des hémocultures, de la ponction lombaire et des prélèvements 

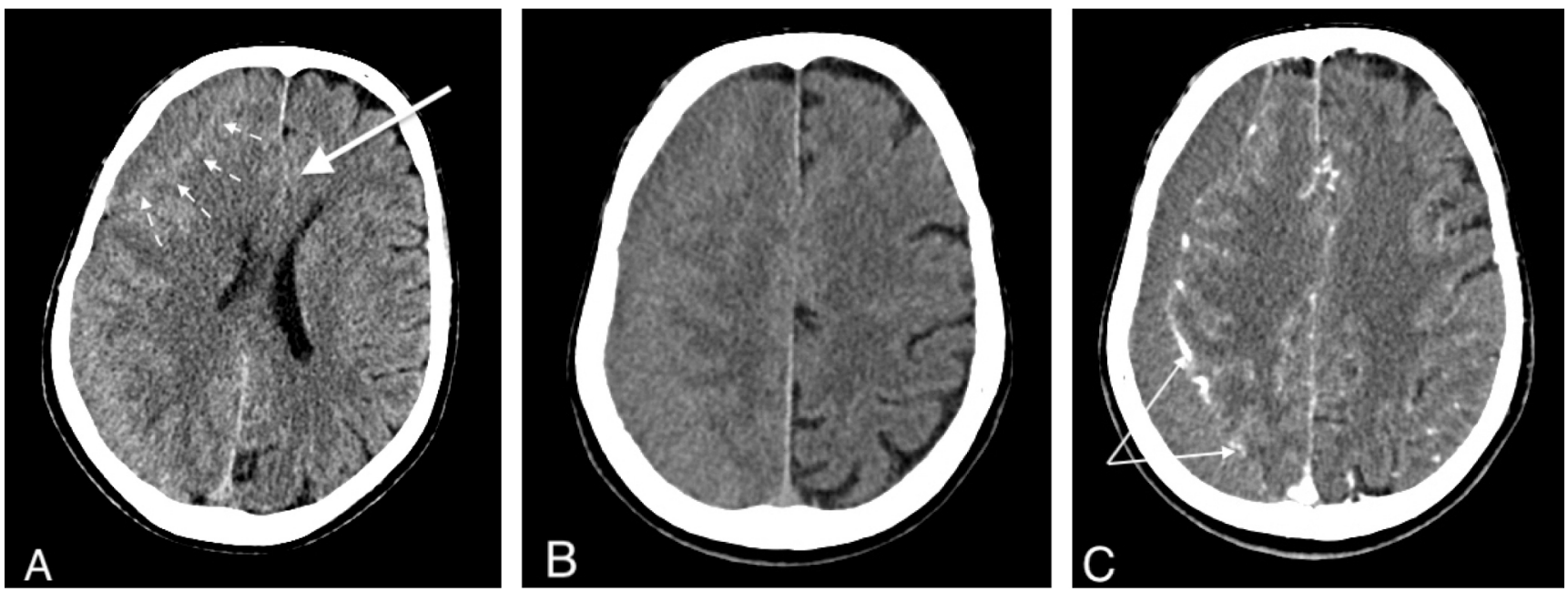

Fig. 1 Scanner cérébral en coupe coronale avant $(A, B)$ et après avec injection de produit de contraste (C). A. Décollement sous-dural frontal (flèches pointillées) et effet de masse avec déviation de la ligne médiane (flèche pleine) ; B. Collection fronto-pariétale droite ; C. Réhaussement de la dure-mère en regard de la collection à l'injection de produit de contraste (flèches)

per opératoires ; d'autre part, une prise en charge neurochirurgicale incontournable. Le recours à une corticothérapie, surtout à visée anti-oedémateuse, est discuté mais n'est pas consensuel selon les experts ; tout comme l'ajout d'un antiépileptique à titre systématique [2], notamment pour le transport du patient vers un centre de neurochirurgie.

\section{Références}

1. Page $C$, Lehmann $P$, Jeanjean $P$ et al (2005) Abcès et empyèmes intracrâniens d'origine ORL. Ann Otolaryngol Chir Cervicofac 122:120-6

2. Mathon B, Korinek AM (2018) L'empyème sous-dural : une urgence neurochirurgicale sous-estimée. Presse Med 47:331-4 